\title{
Implementación de una aplicación informática para la verificación de parámetros de publicación de un artículo científico en revistas indexadas
}

DOI: $\underline{\text { https://doi.org/10.33262/ap.v3i2.42 }}$

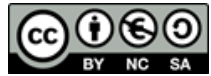

Implementation of a computer application for the verification of publica-tion parameters of a scientific article in indexed journals

\author{
Guerra Poalasin Diego Omar ${ }^{1}$
}

\begin{abstract}
.
Scientific publication, necessary for research and dissemination of information, is immersed in an excess of errors in the review and correction process, whether it is carried out by the researcher or the indexed journal to be published. More demand in research production, greater dissemination of the work and more need for recognition and personal promotion, are parameters that seem to condition the work of many scientists, pushing them towards the slippery slope of fraud. This work aims to create a web application that allows validating and recognizing the parameters and guidelines that a scientific publication must have in order to be published in an indexed journal, which may have greater control in the review and publication process of a scientific article, In addition, this project contributes to the strengthening of research through its dissemination, which is the main mission of the Digital Science Network, linking with the policies of the National Development Plan, by contributing to the increase in the percentage of investment in $R \& D$ of the private sector and contribute to national patent applications by properly completing the books and research raised by the network. Technically, the agile software development methodology (scrum) was used, in the administrative part it is intended to use a garage methodology. This project is focused on the national market in the beginning and the international market in subsequent
\end{abstract}

${ }^{1}$ Ciencia Digital Editorial, Ecuador, diegoguerra@cienciadigital.org 
phases by presenting an automated and validated form to be implemented in an indexed journal in an agile and intelligent way.

Keywords: Publication, application, computing, validation, criteria, articles, indexed magazine

\section{Resumen.}

La publicación científica, necesaria para la investigación y divulgación de información, se encuentra inmersa en un exceso de errores en el proceso de revisión y corrección, ya sea esta realizada por parte del investigador o de la revista indexada a publicar. Más exigencia en la producción investigadora, mayor difusión de los trabajos y más necesidad de reconocimiento y promoción personales, son parámetros que parecen condicionar el trabajo de muchos científicos empujándolos hacia la resbaladiza pendiente del fraude. Este trabajo pretende realizar un aplicativo web que permita validar y reconocer los parámetros y lineamientos que debe tener una publicación científica para poder ser publicada en una revista indexada, lo cual permitirá tener un mayor control en el proceso de revisión y publicación de un artículo científico, además este proyecto contribuirá con el fortalecimiento de la investigación a través de la difusión de la misma que es la misión principal de la Red Ciencia Digital, vinculándose con las políticas del Plan Nacional de Desarrollo, al contribuir con el incremento en el porcentaje de inversión en $\mathrm{I}+\mathrm{D}$ del sector privado y contribuir a las solicitudes de patentes nacionales al culminar adecuadamente los libros e investigaciones planteadas por la red. Técnicamente se usó la metodología de desarrollo ágil de software (scrum), en la parte administrativa se pretende utilizar una metodología de garaje. El presente proyecto está enfocado en el mercado nacional en un inicio y al internacional en fases subsecuentes al presentar un formulario automatizado y validado para ser implementado en una revista indexada de manera ágil e inteligente.

Palabras claves: Publicación, aplicación, informático, validación, criterios, artículos, revista indexadas

\section{Introducción}

El ritmo vertiginoso en los progresos biomédicos, y la gran cantidad de equipos y líneas de investigación existentes, hace necesaria la publicación puntual y controlada, en su calidad y veracidad, de los resultados de estos trabajos, ofreciendo una información indispensable para el desarrollo de nuevas estrategias investigadoras. Asimismo, conocer lo ya investigado y sus conclusiones es indispensable para evitar la duplicidad de proyectos y esfuerzos, especialmente si se tiene en cuenta la gran producción científica actual. No puede concebirse, pues, la investigación sin el soporte de la publicación científica. (Rennie, 1998) 
Según (Miyahira Arakak, 1995) cuando se habla de "publicación científica" suele referirse al mundo de las revistas científicas. Estas tienen ciertas características que no es momento de detallarlas, pero una de ellas es que están respaldadas por cuerpos editoriales que mediante el proceso denominado arbitraje editorial o revisión científica "garantizan" estándares mínimos de calidad de lo que se publica en sus páginas. Los libros sólo pasan ese filtro cuando los respalda alguna institución de carácter más o menos científico, lo que no suele ser habitual en el caso de muchos de los textos utilizados en los cursos de las escuelas o facultades universitarias. Algo similar se puede decir de los resúmenes de trabajos de investigación presentados o publicados en los congresos.

El "arbitraje científico", o peer review para los anglosajones, expresión que podría traducirse como "revisión crítica de los resultados de un artículo científico a cargo de sus pares", abarca la actividad de revisores y editores y se ocupa de determinar si un trabajo merece o no ser publicado, financiado o admitido en una conferencia arbitrada. Siendo así, la condición de creación científica alcanza la condición de proceso social, no solo individual. (Silva AyÇaguer, 2011)

La investigación científica es de vital importancia en el ámbito académico y laboral por ende la revisión realizada por árbitros o revisores es indispensable para la publicación de un artículo, además le aportan al mismo un sello de calidad y seguridad puesto que se verifica su procedencia y desarrollo al igual que parámetros de aceptación impuestos por la revista indexada elegida.

Sin embargo, el proceso de revisión por expertos no ha sido la forma habitual de evaluar artículos científicos. Antes de la segunda guerra mundial, el editor decidía sobre la aceptabilidad de los manuscritos, generalmente sin la ayuda de revisores externos. Pero, a partir de los años cuarenta, la capacidad (y credibilidad) de los editores para ser árbitros universales se vió rápidamente limitada por el aumento de las especialidades médicas. Hoy en día, el proceso de revisión editorial por expertos es reconocido como la norma para la definición de una revista de calidad en cualquier disciplina científica. De hecho, este proceso de revisión ha cobrado tanta importancia que en medios universitarios de EEUU por ejemplo, la capacidad de investigador para publicar en revistas donde se utiliza el sistema de revisión editorial constituye uno de los aspectos más importantes en el currículum vitae de un candidato a un puesto o una promoción académica. (Szklo \& Nieto , 1993)

A los árbitros les toca evaluar si las preguntas de investigación o los propósitos de un ensayo son pertinentes; si la metodología seguida es correcta y si los resultados constituyen un aporte a lo que ya se conoce; si los autores se han apoyado en las referencias correctas y, en buena medida, si la obra responde a las normas éticas establecidas por la revista. No se espera del sistema, sin embargo, que tenga capacidad para detectar fraudes. (Silva AyÇaguer, 2011) 
Para varias revistas digitales el contar con un aplicativo informático, que les permita a los árbitros o revisores realizar la revisión de artículos de una forma sencilla e intuitiva es fundamental en el mundo actual, la escasez de plataformas que permitan hacer esta actividad es notoria lo cual dificulta el libre desarrollo y realización de las revistas digital actualmente.

(Wordpress, 2000) Permite crear un sitio web que satisfaga las necesidades específicas de la. Además permite crear un blog, un sitio profesional, un portafolio, una tienda en línea o cualquier cosa que se pueda imaginar. Con una optimización integrada y temas adaptables para móviles, con la cual se puede llegar a tantos usuarios como se necesite.

El objetivo de la presente investigación fue; realizar la implementación de una aplicación informática en forma de plugin (complemento para wordpress), que sirva de apoyo para la revisión y validación de artículos científicos en base a parámetros como; número de hojas, numero de palabras, bibliografía entre otros aspectos importantes al momento de realizar un artículo y publicarlo dentro de revistas digitales tanto internacionales como nacionales.

\section{Metodología}

La presente investigación se realizó bajo las siguientes modalidades:

Modalidad Bibliográfica o Documentada: La investigación fue bibliográfica porque permitió ampliar y profundizar diferentes enfoques, teorías, conceptualizaciones y criterios de diversos autores sobre el tema de estudio.

Además la investigación fue experimental porque se intentó buscar la mejor solución que se adapte a las necesidades y requisitos que exigía la presente investigación.

\section{Herramientas e instrumentos de desarrollo}

- Wordpress

Es un gestor de contenidos basados en la creación de blog o bitácoras web el cual está enfocado a la creación y personalización de una página web que permite un alojamiento gratuito o de pago.

\section{- PHP (acrónimo recursivo de PHP: Hypertext Preprocessor)}

Es un lenguaje de código abierto muy popular especialmente adecuado para el desarrollo web y que puede ser incrustado en HTML. Lo que distingue a PHP de algo del lado del cliente como Javascript es que el código es ejecutado en el servidor, generando HTML y enviándolo al cliente. El cliente recibirá el resultado de ejecutar el script, aunque no se sabrá el código subyacente que era. El servidor web puede ser configurado incluso para que procese todos los ficheros HTML con PHP, por lo que 
no hay manera de que los usuarios puedan saber qué se tiene debajo de la manga. (Php, 2001)

\section{- JavaScript}

Es un lenguaje de programación que te permite realizar actividades complejas en una página web - cada vez más una página web hace más cosas que sólo mostrar información estática - como mostrar actualizaciones de contenido en el momento, interactuar con mapas, animaciones gráficas 2D/3D etc. — puedes estar seguro que JavaScript está involucrado. Es la tercera capa del pastel de los estándares en las tecnologías para la web, dos de las cuales son (HTML y CSS), hablaremos de ellas más adelante con más detalle en otra parte de nuestra Área de Aprendizaje. (Docs, Developer mozilla, 2019)

- CSS

Hojas de Estilo en Cascada (del inglés Cascading Style Sheets) o CSS es el lenguaje utilizado para describir la presentación de documentos HTML o XML, esto incluye varios lenguajes basados en XML como son XHTML o SVG. CSS describe como debe ser renderizado el elemento estructurado en pantalla, en papel, hablado o en otros medios. (Docs, Developer mozilla, 2019)

\section{Recolección de la Información}

Para la recolección de información se utilizó la técnica de investigación documental de tipo informativa, obteniendo información relevante de diferentes fuentes confiables, además de páginas de revistas indexadas y editoriales digitales, analizando y seleccionando la información de mayor relevancia la cual sirvió de apoyo para el desarrollo de esta investigación.

\section{Procesamiento y análisis de la información}

Para el, procesamiento y análisis de la información se aplicó los siguientes procedimientos

- Recolección de información sobre los procesos requeridos para la generación y validación de un artículo científico.

- Análisis de la información obtenida.

- Interpretación de la información obtenida

- Diseño de proceso

- Desarrollo del aplicativo.

\section{Desarrollo del proyecto}

- Recolección de requerimientos para el aplicativo 
- Diseño de la base de datos para la aplicación

- Diseño de la interfaz del aplicativo

- Desarrollo y programación del aplicativo

- Pruebas de validación y funcionamiento del aplicativo.

\section{Resultados}

La aplicación planteada para el desarrollo del arbitraje de cada artículo contendrá 4 módulos principales los cuales son:

- Realizar arbitraje: Modulo de ingreso de los datos del artículo (Fecha de revisión, Revista de publicación y titulo del artículo), y listado de preguntas a responder.

- Gestionar preguntas: Modulo de gestión de preguntas del arbitraje, edición y eliminación de las preguntas.

- Adicionar preguntas: Modulo de creación generación de nuevas preguntas.

- Arbitrajes respondidos: Modulo donde se encuentran el listado de arbitrajes respondidos, en el cual se puede descargar el formulario de arbitraje respondido en formato Word, pdf y html.

El funcionamiento del aplicativo web dentro de la plataforma wordpress se maneja en un esquema de base de datos el cual, guarda la información de revisión de cada artículo (Numero, revisor, preguntas, fecha, tema del artículo), esta base de datos se la realizo en MYSQL con la siguiente estructura (Ver imagen).

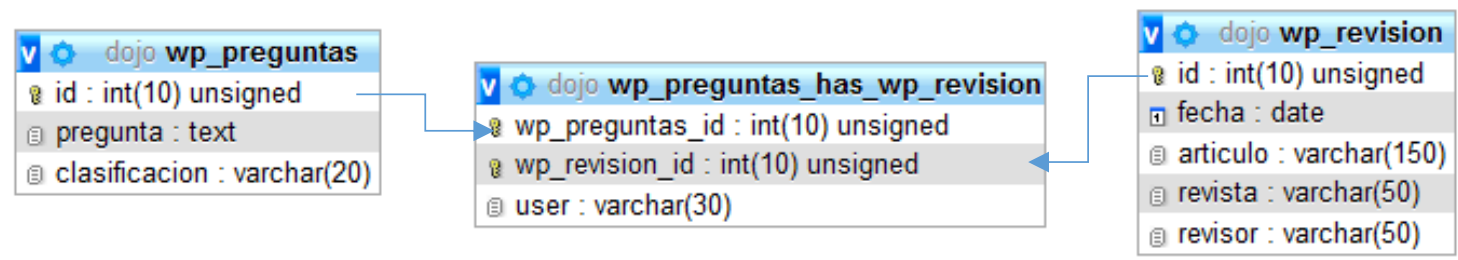

Figura 1; Esquema base de datos para el formulario de revisión Elaborado por; Autores

En el desarrollo del formulario de arbitraje, lo primero que se realizo es formatear la plantilla del formato del artículo científico en formato odt como se muestra en la figura. 


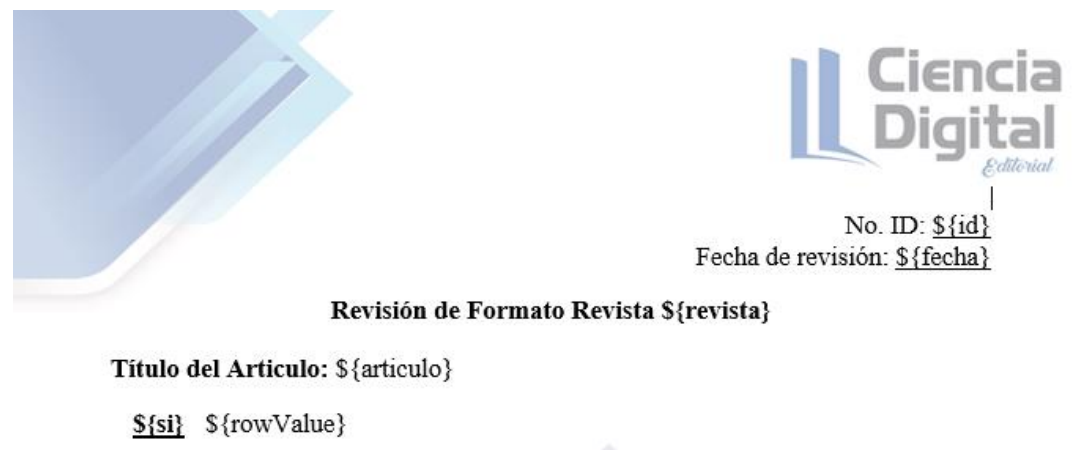

Figura 2; Plantilla formateada formato de revisión articulo Elaborado por; Autores

De igual manera para la generación del documento de revisión en formato pdf se procedió a realizar un archivo PHP el cual contiene la estructura del formato de revisión como se muestra en la figura.

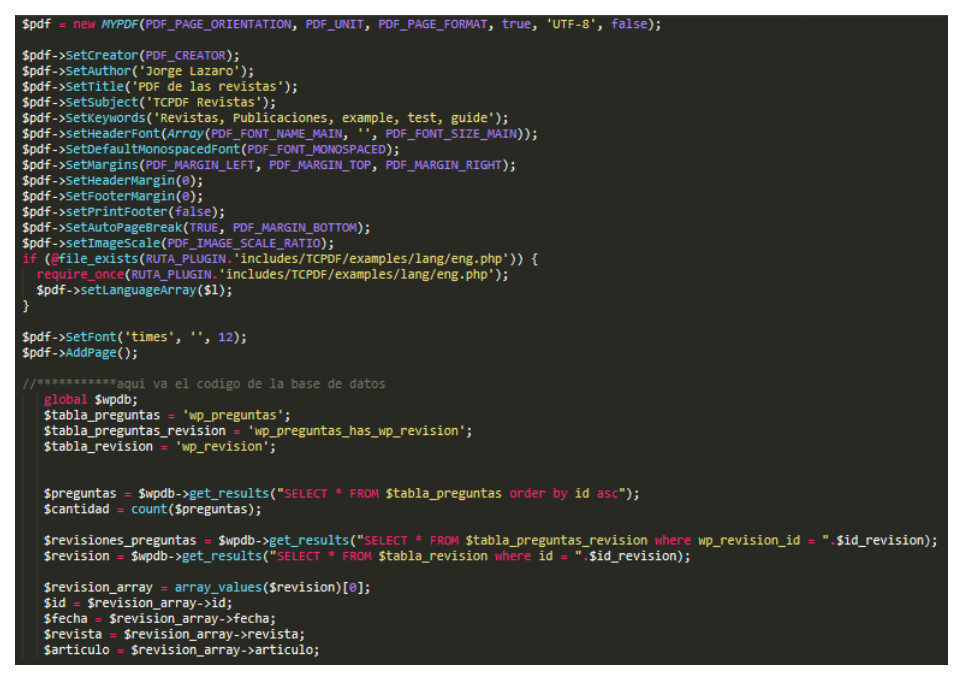

Figura 3; Archivo PHP de configuración del formato de revisión articulo Elaborado por; Autores

\section{Preguntas arbitraje}

La revisión y validación de cada artículo se lo aplica respondiendo una serie de preguntas las cuales deben ser respondidas en un $75 \%$ para verificar que un artículo es válido para su publicación, dependiendo de cada revista indexada este porcentaje puede variar según los lineamientos o esquemas que tenga. 
Las preguntas realizadas en el arbitraje tienen una clasificación de importancia las cuales se las califica dependiendo de la necesidad de cada revista, la escala de importancia es de la siguiente manera:

- Importante

- Intermedio

- Opcional

La lista de preguntas tiene un numero de 105 preguntas entre importantes intermedias y opcionales que, dependiendo de la observación de cada revisor las calificara con respecto al artículo planteado.

Tabla 1: Preguntas de arbitraje

\begin{tabular}{|r|l|}
\hline 1 & $\begin{array}{l}\text { El Resumen sigue la estructura indicada en las Normas de la Revista: objetivo, } \\
\text { antecedentes (opcional), metodología, resultados, y conclusiones }\end{array}$ \\
\hline 2 & $\begin{array}{l}\text { El Resumen es un solo párrafo y no supera las 10-11 líneas (aproximadamente } \\
150-200 \text { palabras) }\end{array}$ \\
\hline 3 & El Resumen no contiene citas a la literatura \\
\hline 4 & $\begin{array}{l}\text { La Introducción está bien documentada, con suficientes citas a literatura de } \\
\text { Corriente Principal (incluidas en WoS, Scopus, Scielo) }\end{array}$ \\
\hline 5 & $\begin{array}{l}\text { Las citas a la literatura incluyen al menos 2/3 de citas a literatura de corriente } \\
\text { principal (WoS, Scopus, Scielo) }\end{array}$ \\
\hline 6 & $\begin{array}{l}\text { En el listado de referencias, las que son de revistas de corriente principal en inglés, } \\
\text { están con letras de color rojo }\end{array}$ \\
\hline 7 & $\begin{array}{l}\text { Las que correspondan a revistas de corriente principal en castellano están con } \\
\text { letras de color azul }\end{array}$ \\
\hline 8 & El resto de las referencias, de cualquier otro tipo están con letras de color negro \\
\hline 9 & $\begin{array}{l}\text { El trabajo incluye una sección corta de \"Conclusionesl", siguiendo normas } \\
\text { internacionales }\end{array}$ \\
\hline 10 & $\begin{array}{l}\text { La sección de Conclusiones incluye solo “conclusiones", no otros aspectos que } \\
\text { deben ir en otra parte, como discusiones o resultados. }\end{array}$ \\
\hline 11 & $\begin{array}{l}\text { La cantidad de cifras significativas y decimales usadas en el artículo son adecuadas } \\
\text { y uniformes }\end{array}$ \\
\hline 12 & $\begin{array}{l}\text { Se usa notación estándar (comúnmente usada en la literatura) para variables y } \\
\text { propiedades }\end{array}$ \\
\hline 13 & $\begin{array}{l}\text { En el trabajo (incluidos el Resumen y el título) no se usa abreviaturas innecesarias } \\
\text { o poco conocidas }\end{array}$ \\
\hline 14 & $\begin{array}{l}\text { No se usan palabras extranjeras cuando ya existe una en castellano (o puedo } \\
\text { acuñar una) }\end{array}$ \\
\hline 15 & $\begin{array}{l}\text { He revisado el texto para que esté libre de errores de ortografía, puntuación, } \\
\text { estructura y redacción }\end{array}$ \\
\hline
\end{tabular}




\begin{tabular}{|c|c|}
\hline 16 & $\begin{array}{l}\text { En ninguna parte se usa el barbarismo "estado del arte" } \\
\text { (http://www.scielo.cl/pdf/formuniv/v2n3/art01.pdf) }\end{array}$ \\
\hline 17 & No hay títulos de secciones y subsecciones seguidas (sin texto entre ellos) \\
\hline 18 & $\begin{array}{l}\text { Las páginas tienen el tamaño correcto (A4 de } 21 \times 29.7 \mathrm{~cm} \text { ) y márgenes de } 2 \mathrm{~cm} \text {. en } \\
\text { todos los lados, y en diseño con encabezado y pie de página de } 1 \mathrm{~cm} \text {. }\end{array}$ \\
\hline 19 & El artículo, en el formato de la revista, tiene al menos 8 páginas completas \\
\hline 20 & $\begin{array}{l}\text { Si el trabajo tiene } 8 \text { páginas, la última, la octava, está ocupada al menos en tres } \\
\text { cuartos de página }\end{array}$ \\
\hline 21 & $\begin{array}{l}\text { Solamente el título del artículo (en inglés y castellano) y los títulos de secciones } \\
\text { están con negritas }\end{array}$ \\
\hline 22 & $\begin{array}{l}\text { Todo el texto (excepto tablas, figuras y el título (en inglés y castellano) está con } \\
\text { letra arial } 10\end{array}$ \\
\hline 23 & $\begin{array}{l}\text { Todos los párrafos están ajustados a ambos lados (justified) y sin ningún tipo de } \\
\text { sangría ni viñetas }\end{array}$ \\
\hline 24 & La separación entre párrafos es de un espacio simple (un espacio sencillo en Word) \\
\hline 25 & $\begin{array}{l}\begin{array}{l}\text { El texto lleva separación simple (sencilla) entre líneas (esto es, a renglón seguido, } \\
\text { interlineado sencillo) }\end{array} \\
\end{array}$ \\
\hline 26 & No hay sangrías, ni viñetas, ni subrayados en todo el trabajo \\
\hline 27 & Los títulos de secciones o subsecciones no están numerados \\
\hline 28 & No hay marcaciones automáticas dirigidas a pie de página o a otro lugar en el texto \\
\hline 29 & El trabajo en formato Word y en PDF tienen tamaños inferiores a $2 \mathrm{Mb}$ cada uno \\
\hline 30 & $\begin{array}{l}\text { No hay abuso en la separación de párrafos } \\
\text { (http://bvs.sld.cu/revistas/san/vol11_1_07/san06107.htm) }\end{array}$ \\
\hline 31 & $\begin{array}{l}\text { No se proponen trabajos futuros sobre el tema ni se describe en que están } \\
\text { trabajando los autores }\end{array}$ \\
\hline 32 & $\begin{array}{l}\text { La primera página sigue el formato (estructura, tipo de letra y puntuación) que se } \\
\text { muestra al final de esta Tabla }\end{array}$ \\
\hline 33 & $\begin{array}{l}\text { Los nombres de los autores van seguidos en la línea, separados por comas, con } \\
\text { letras arial } 10 \text { en negritas. Como en este ejemplo, pero con arial } 10 \text { en negritas: } \\
\text { Jorge A. Autoruno, Daniel M. Autordos y Pedro Autortres }\end{array}$ \\
\hline 34 & $\begin{array}{l}\text { Cada autor tiene una sola afiliación (la principal y máximo una más si es } \\
\text { necesario) }\end{array}$ \\
\hline 35 & $\begin{array}{l}\text { Los nombres de los autores están en formato USA: 1er nombre, inicial del } 2^{\circ} \\
\text { nombre, apellido principal }\end{array}$ \\
\hline 36 & $\begin{array}{l}\text { Si un autor usa dos apellidos estos vienen conectados por un guion (por ejemplo } \\
\text { (Pérez-Araya) }\end{array}$ \\
\hline 37 & Las direcciones de correo-e en la afiliación de los autores y autoras están correctas \\
\hline 38 & El título del trabajo tiene como máximo tres líneas cuando se escribe con arial 16 \\
\hline 39 & $\begin{array}{l}\text { El título del trabajo está con mayúscula en las palabras principales y sin punto al } \\
\text { final (Ejemplo: Modelado de la Extracción de Aceite de Clavo de Olor mediante } \\
\text { Elementos Finitos usando Algor Windows) }\end{array}$ \\
\hline
\end{tabular}




\begin{tabular}{|c|c|}
\hline 40 & $\begin{array}{l}\text { La primera página lleva título en inglés (traducción correcta del título en } \\
\text { Castellano) (Ejemplo: Modeling of the Extraction of Clove Oil by Finite Elements } \\
\text { using Algor Windows) }\end{array}$ \\
\hline 41 & $\begin{array}{l}\text { La primera página lleva la versión en inglés del resumen (correcta traducción, de } \\
\text { preferencia inglés estadounidense, pero se acepta inglés británico) }\end{array}$ \\
\hline 42 & $\begin{array}{l}\text { Las palabras clave están en cursiva, separadas por; (punto y coma) y con } \\
\text { minúsculas, (excepto nombres propios y siglas) (Palabras clave: agua de mar; } \\
\text { soluciones salinas; } \mathrm{NaCl} \text {; viscosidad; densidad; redes neuronales) }\end{array}$ \\
\hline 43 & $\begin{array}{l}\text { Las keywords son las mismas y están en el mismo formato que las palabras clave } \\
\text { en Castellano (Keywords: seawater, saline solutions, NaCl; viscosity; density; } \\
\text { neural networks) }\end{array}$ \\
\hline 44 & Las ecuaciones son editables con editores de ecuaciones estándares \\
\hline 45 & $\begin{array}{l}\text { Las ecuaciones y la numeración de ellas están en tablas (de dos columnas), con } \\
\text { bordes ocultos }\end{array}$ \\
\hline 46 & $\begin{array}{l}\text { Todas las ecuaciones están con arial (tamaño } 9 \text { o 10), ajustadas a la izquierda (en } \\
\text { la celda izquierda) }\end{array}$ \\
\hline 47 & $\begin{array}{l}\begin{array}{l}\text { Los números de ecuaciones están en paréntesis redondos y ajustados a la derecha } \\
\text { (en la celda derecha) }\end{array}\end{array}$ \\
\hline 48 & No hay ecuaciones incrustadas en el texto. \\
\hline 49 & La sección de Referencias es la última sección del trabajo \\
\hline 50 & $\begin{array}{l}\text { Hay suficientes citas a artículos clásicos y de los últimos años publicados en } \\
\text { revistas de corriente principal }\end{array}$ \\
\hline 51 & $\begin{array}{l}\text { Las Referencias llevan una separación de } 6 \text { puntos entre una y otra (no van a } \\
\text { renglón seguido) }\end{array}$ \\
\hline 52 & $\begin{array}{l}\text { El artículo tiene menos de } 30 \text { referencias, establecidas como máximo por la } \\
\text { revista. }\end{array}$ \\
\hline 53 & $\begin{array}{l}\text { El artículo tiene más de } 30 \text { referencias, porque se trata de un artículo tipo } \\
\text { "Review" (ya conversado con el Editor) }\end{array}$ \\
\hline 54 & $\mathrm{Al}$ menos $2 / 3$ de las referencias son a revistas y libros de corriente principal. \\
\hline 55 & Al menos la mitad de todas las referencias se refieren a documentos en inglés \\
\hline 56 & $\begin{array}{l}\text { Las citas a la literatura están por autor y año (Arias, 2007; Pérez y Rojas, 2006; } \\
\text { Pérez et al., 2005) }\end{array}$ \\
\hline 57 & $\begin{array}{l}\text { La lista de documentos citados se titula \"REFERENCIAS\" y no Bibliografía o } \\
\text { similares }\end{array}$ \\
\hline 58 & Las referencias están en orden alfabético, por apellido del 1er autor \\
\hline 59 & Las referencias están sin numeración, sangría, guiones, ni ningún otro símbolo \\
\hline 60 & Todas las referencias terminan con el año entre paréntesis redondos \\
\hline 61 & $\begin{array}{l}\text { Las referencias están completas, como para poder llegar a ellas en forma } \\
\text { inequívoca a cada una de ellas, y siguen las Normas en todos sus aspectos }\end{array}$ \\
\hline 62 & $\begin{array}{l}\text { Las referencias a artículos de revistas con menos de } 4 \text { autores siguen este formato: } \\
\text { Paz, H.L., Melo, N.N. y Castro, J.L., Datos Experimentales de Lubricantes, Int. J. } \\
\text { Lubrication, 22(4), 123-131 (2003) }\end{array}$ \\
\hline
\end{tabular}




\begin{tabular}{|c|c|}
\hline 63 & $\begin{array}{l}\text { Las referencias a artículos de revistas con más de } 4 \text { autores siguen este formato: } \\
\text { Smith, J.L., Gray, J. y otros ocho autores, Observations of the Celestial Movement, } \\
\text { J. Weird Physics , 13(4),23-34(2006) }\end{array}$ \\
\hline 64 & $\begin{array}{l}\text { Las referencias a libros siguen este formato: Stiles, J.P., Handbook of Non- } \\
\text { Conventional Energy, } 2^{\mathrm{a}} \text { edición, 23-58. Brooks Publishers, Londres, Inglaterra } \\
(1990)\end{array}$ \\
\hline 65 & $\begin{array}{l}\text { Las referencias a artículos de Revistas electrónicas (con DOI) siguen este formato: } \\
\text { Torres, L.J., Extracción de Aceites Esenciales de Frutos Cítricos, doi: } \\
\text { 10.4067/S0718-07642014000400008, Inf. Tecnol., 37(3), 45-56 (2004) Wolf, J.K. } \\
\text { y Wall, K.L., Phase Equilibria in Ternary Alcoholic Mixtures, doi: } \\
\text { 12.016/j.equilibrium.2012.01.0303, J. of Equilibrium, 41(6), 37-45 (2013) }\end{array}$ \\
\hline 66 & $\begin{array}{l}\text { Las referencias a artículos de Revistas electrónicas (sin DOI) siguen este formato: } \\
\text { Torres, L.J., Caracterización Reológica de Pastas de Jamón, Rev. Ciencia, ISSN: } \\
\text { 0655-6782, 37(3), 34-37, (2003) }\end{array}$ \\
\hline 67 & $\begin{array}{l}\text { Para otros tipos de documentos he revisado las normas de la revista y las he } \\
\text { seguido }\end{array}$ \\
\hline 68 & Toda referencia aparece citada en el trabajo \\
\hline 69 & Toda cita en el trabajo tiene su referencia correcta en el listado de referencias \\
\hline 70 & $\begin{array}{l}\text { La forma, estructura, puntuación, y ortografía de los nombres de los autores en el } \\
\text { listado de referencias y la cita en el cuerpo del artículo son iguales (vi el punto } \\
23.13 \text { en Normas, por detalles y ejemplos). }\end{array}$ \\
\hline 71 & $\begin{array}{l}\text { Las leyendas de los ejes de las figuras están con tamaño adecuado y proporcional a } \\
\text { la figura (arial } 8 \text { o 9) }\end{array}$ \\
\hline 72 & $\begin{array}{l}\text { Los ejes de las figuras no están recargados de números y el interior no está } \\
\text { recargado de grillas (líneas) }\end{array}$ \\
\hline 73 & $\begin{array}{l}\text { Los números en los ejes y en el interior de las figuras están con la cantidad de } \\
\text { decimales adecuada }\end{array}$ \\
\hline 74 & $\begin{array}{l}\text { Todas las figuras están en escala de grises o colores distinguibles cuando se } \\
\text { imprime en escala de grises }\end{array}$ \\
\hline 75 & Las figuras son claras y legibles, con letras y números adecuados si los hay \\
\hline 76 & $\begin{array}{l}\text { Las figuras no están incrustadas. Esto es que cuando el texto se mueve, por } \\
\text { ejemplo si continúo escribiendo, las figuras también deben moverse con el texto. }\end{array}$ \\
\hline 77 & Las leyendas de Tablas y Figuras son con arial 9, sin negritas ni cursivas \\
\hline 78 & $\begin{array}{l}\text { Las tablas y figuras se citan en el texto como: Tabla x: Figura x; Fig. x (x es el } \\
\text { número que corresponda) }\end{array}$ \\
\hline 79 & En el interior de Tablas no hay celdas con fondo de colores y de ningún tipo \\
\hline 80 & Las leyendas internas en Figuras tienen tamaño 9 y no se usa letras en negritas \\
\hline 81 & $\begin{array}{l}\text { Las Figuras no llevan títulos interiores (eso va en la leyenda, en la parte inferior de } \\
\text { la figura) }\end{array}$ \\
\hline 82 & Las leyendas del eje vertical van en forma vertical (girada en $90^{\circ}$ ) \\
\hline 83 & No hay "notas al pie" en páginas, en figuras, ni en tablas \\
\hline 84 & $\begin{array}{l}\text { Las Figuras están designadas y numeradas como \"Fig. x: \", seguidas de una } \\
\text { adecuada leyenda }\end{array}$ \\
\hline
\end{tabular}




\begin{tabular}{|c|c|}
\hline 85 & $\begin{array}{l}\text { Las Tablas están designadas y numeradas como \"Tabla x: \", seguidas de una } \\
\text { adecuada leyenda }\end{array}$ \\
\hline 86 & Las leyendas de Figuras van en la parte inferior de la Figura, centrada a la figura \\
\hline 87 & $\begin{array}{l}\text { Si las leyendas de Figuras ocupan más de una línea, van ajustadas a la izquierda de } \\
\text { la figura }\end{array}$ \\
\hline 88 & Las leyendas de Tablas van en la parte superior de la Tabla, centrada a la tabla \\
\hline 89 & $\begin{array}{l}\text { Si las leyendas de Tablas ocupan más de una línea van ajustadas a la izquierda de } \\
\text { la tabla }\end{array}$ \\
\hline 90 & El grosor de las líneas en figuras y tablas es similar al de las letras del texto \\
\hline 91 & $\begin{array}{l}\text { Todas las figuras son editables (que el editor las pueda editar, intervenir, si es } \\
\text { necesario) }\end{array}$ \\
\hline 92 & $\begin{array}{l}\text { Las Tablas son mencionadas en el texto por su número: no se usa, "la tabla } \\
\text { siguiente" o "la tabla de abajo" }\end{array}$ \\
\hline 93 & $\begin{array}{l}\text { Las Figuras son mencionadas en el texto por su número (no se usa, "la figura } \\
\text { siguiente" o "la figura de abajo") }\end{array}$ \\
\hline 94 & $\begin{array}{l}\text { Las Figuras tomadas de otras fuentes deben llevar autorización (Reproducida con } \\
\text { autorización de...) }\end{array}$ \\
\hline 95 & $\begin{array}{l}\text { Las Figuras adaptadas de otras fuentes deben llevar el crédito correspondiente } \\
\text { (p.ej., Adaptada de Perez, 2013) }\end{array}$ \\
\hline 96 & $\begin{array}{l}\text { No hay "pie de tablas" ni "pie de figuras". Todo lo necesario va en la leyenda de } \\
\text { las tablas o en el texto }\end{array}$ \\
\hline 97 & $\begin{array}{l}\text { En figuras y tablas no se coloca la sentencia "Fuente: elaboración propia", porque } \\
\text { no es necesario }\end{array}$ \\
\hline 98 & $\begin{array}{l}\text { Cuando se cita una figura se escribe "en la figura x se muestra" y no "en la } \\
\text { siguiente figura", por ejemplo }\end{array}$ \\
\hline 99 & $\begin{array}{l}\text { Cuando se cita una tabla se escribe "en la tabla x se muestra" y no "en la siguiente } \\
\text { tabla", por ejemplo }\end{array}$ \\
\hline 100 & $\begin{array}{l}\text { Si una tabla debe ser dividida en dos páginas la segunda página ocupada lleva } \\
\text { como leyenda el número de la Tabla seguida de (continuación): Ejemplo: Tabla } 3 \\
\text { (continuación) }\end{array}$ \\
\hline 101 & $\begin{array}{l}\text { Las abreviaturas del nombre de las revistas en el listado de referencias, están } \\
\text { escritas como lo indica la revista que está citando }\end{array}$ \\
\hline 102 & $\begin{array}{l}\text { El DOI de la revista en el listado de referencias, está escrito como lo indica la } \\
\text { revista que está citando }\end{array}$ \\
\hline 103 & $\begin{array}{l}\text { El título del artículo y de la revista en el listado de referencias, va con mayúscula } \\
\text { en cada palabra principal, como los ejemplos de los numerales } 62 \text { a } 66\end{array}$ \\
\hline 104 & $\begin{array}{l}\text { Las Referencias están con letra arial } 9 \text { y separadas entre ellas por } 6 \text { ptos (ptos. } \\
\text { como lo define WORD) }\end{array}$ \\
\hline 105 & $\begin{array}{l}\text { Las Referencias no llevan ningún tipo de marcación o enlace y si he usado } \\
\text { Mendeley u otros gestores de referencias, el listado final incluido esta como texto. }\end{array}$ \\
\hline
\end{tabular}

Elaborado por; Autores 


\section{Diseño de interfaces}

\section{Realizar arbitraje}

Ventana en el cual se realiza el arbitraje ingresando los datos requeridos.

\begin{tabular}{|c|c|c|}
\hline & Revisión de Formato Para la Revista seleccionada & \\
\hline Fecha de tev & & \\
\hline $2020-11$ & & 国 \\
\hline Revista de la & ubicación & \\
\hline Anatomi & Digital & ऽ \\
\hline Thulo del ant: & & \\
\hline Anatom & humana & \\
\hline Clasificaci & in (C): Importante (I), Intermedio (M), Opcional (O) & \\
\hline$\square$ Marc & ar/Desmarcar Todos & \\
\hline (C) & Listado de Preguntas. & \\
\hline$\square$ (I) & El Resumen sigue la estructura indicada en las Normas de la Revista: objetivo, antecedentes (opcional), metodología, resultados, y conclusiones & \\
\hline$\square$ (l) & El Resumen es un solo párrafo y no supera las 10-11 lineas (aproximadamente 150-200 palabras) & \\
\hline$\square(\mathrm{M})$ & El Resumen no contiene citas a la literatura & \\
\hline$\square(0)$ & La Introducción está bien documentada, con suficientes citas a literatura de Corriente Principal (incluidas en WoS, Scopus, Scielo) & \\
\hline$\square$ (l) & Las citas a la literatura incluyen al menos $2 / 3$ de citas a literatura de corriente principal (WoS, Scopus, Scielo) & \\
\hline$\square(0)$ & En el listado de referencias, las que son de revistas de corriente principal en inglés, están con letras de color rojo & \\
\hline$\square(\mathrm{M})$ & Las que correspondan a revistas de corriente principal en castellano están con letras de color azul & \\
\hline$\square$ (I) & El resto de las referencias, de cualquier otro tipo están con letras de color negro & \\
\hline$\square$ (M) & El trabajo incluye una sección corta de |"Conclusiones|", siguiendo normas internacionales & \\
\hline
\end{tabular}

Figura 4; Ventana revisión de formato para revista Elaborado por; Autores

\section{Gestionar preguntas}

Ventana en el cual se gestiona (edición y eliminación) las preguntas ingresadas.

Listado de preguntas

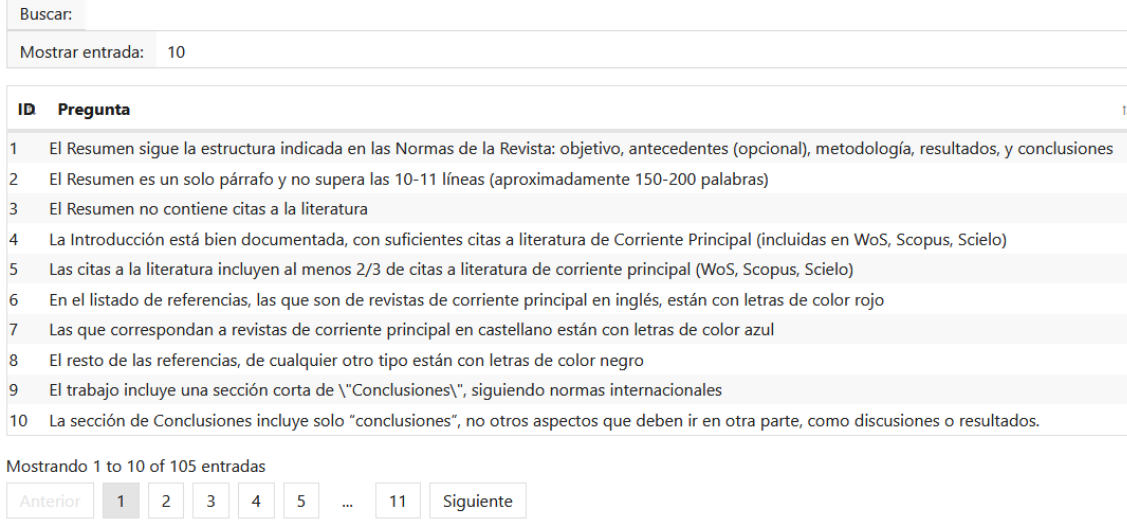

Figura 5; Ventana gestión de preguntas Elaborado por; Autores 


\section{Adicionar preguntas}

Ventana en el cual se crea o adiciona nuevas preguntas al formulario de arbitraje.

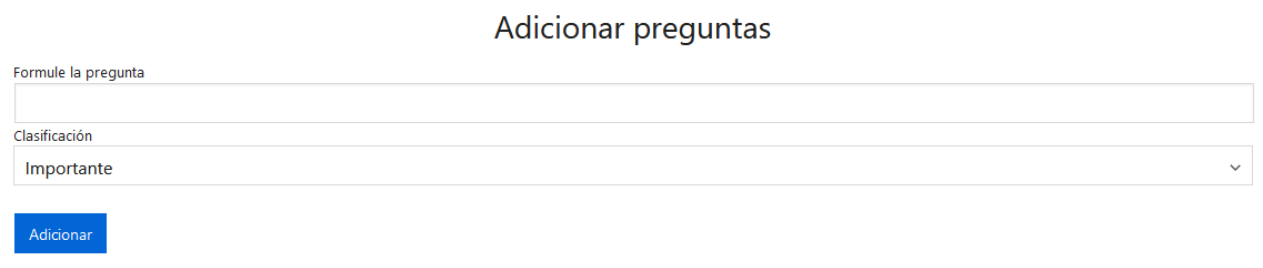

Figura 6; Ventana adición de preguntas

Elaborado por; Autores

\section{Arbitrajes respondidos}

Ventana donde se guardan los arbitrajes respondidos.

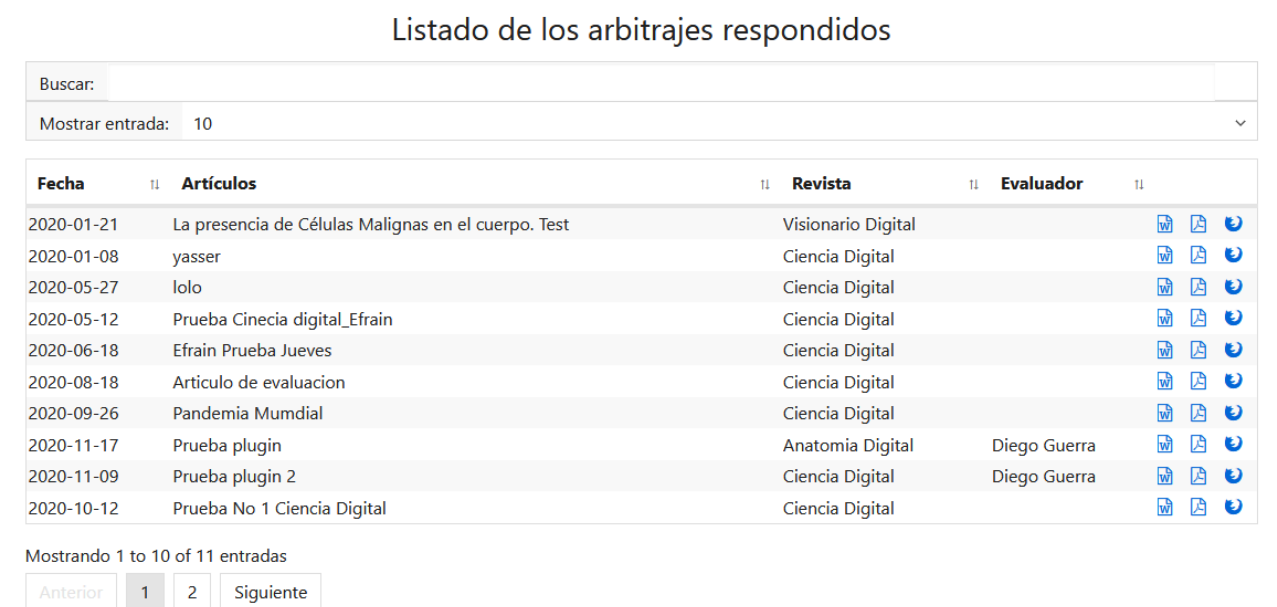

Figura 7; Ventana arbitrajes respondidos

Elaborado por; Autores

Como resultado de los arbitrajes respondidos se obtendrá un archivo en formato doc o pdf, el cual dependiendo de las necesidades del revisor obtendrá el acceso a cada uno. En el formato que se genera constaran los datos ingresados previamente para la realización de arbitraje, al igual del nombre del revisor que realizo el arbitraje, en el documento además se visualizará una estadística del arbitraje en el que consta el número de preguntas respondidas y el peso que tiene cada una. (Ver figura) 

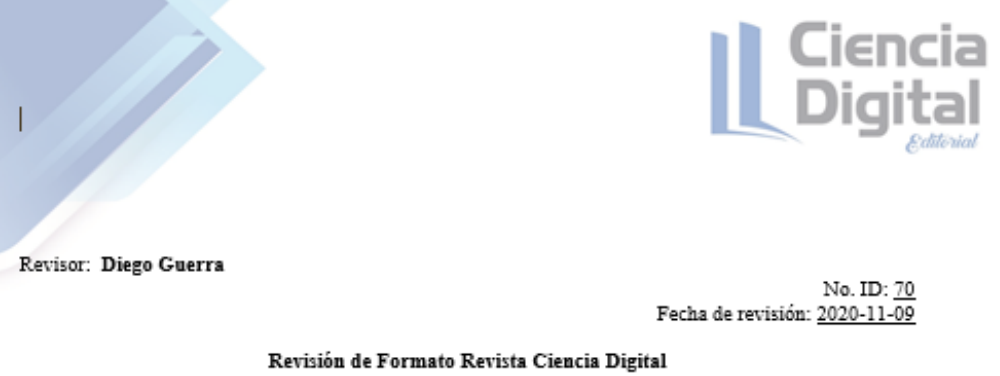

Título del Articulo: Prueba plugin 2

Estadística del artículo que se intenta publicar

\begin{tabular}{|c|c|c|c|}
\hline Clasificación & Cantidad & Respondidos & Porciento \\
\hline Importante & 64 & 64 & 50 \\
\hline Intermedio & 34 & 34 & 30 \\
\hline Opcional & 7 & 7 & 20 \\
\hline & & Total & 100 \\
\hline
\end{tabular}

Respuestas a preguntas

\begin{tabular}{|r|l|l|}
\hline 1 & $\begin{array}{l}\text { El Resumen sigue la estructura indicada en las Normas de la Revista: objetivo, antecedentes } \\
\text { (opcional), metodologia, resultados, y conclusiones }\end{array}$ & SI \\
\hline 2 & El Reaumen es un solo párrafo y no supera las 10-11 lineas (aproximadamente 150-200 palabras) & SI \\
\hline 3 & El Reaumen no contiene citas a la literatura & SI \\
\hline 4 & $\begin{array}{l}\text { La Introducción está bien documentada, con guficientes citas a literatura de Corriente Principal } \\
\text { (incluidas en WoS, Scopus, Scielo) }\end{array}$ & $\underline{\text { SI }}$ \\
\hline 5 & $\begin{array}{l}\text { Las citas a la literatura incluyen al menos 2/3 de citas a literatura de corriente principal (WoS, } \\
\text { Scopus, Scielo) }\end{array}$ & $\underline{\text { SI }}$ \\
\hline 6 & $\begin{array}{l}\text { En el listado de referencias, las que son de revistas de corriente principal en inglés, están con letras } \\
\text { de color rojo }\end{array}$ & $\underline{\text { SI }}$ \\
\hline 7 & Las que correspondan a revistas de corriente principal en castellano están con letras de color azul & $\underline{\text { SI }}$ \\
\hline
\end{tabular}

Figura 8; Documento arbitraje

Elaborado por; Autores

\section{Conclusiones.}

- Mediante la realización de esta investigación se obtuvo una solución óptima y viable para la solución y desarrollo del tema planteado, dando a conocer varios puntos esenciales al tener en cuenta al momento de realizar el arbitraje a un artículo determinado dentro de una revista digital indexada.

- El formulario web realizado permitió controlar todos los parámetros de calificación y revisión de un artículo, lo cual permite llevar de una manera más optima el proceso de revisión y calificación de un artículo.

\section{Referencias bibliográficas.}

Docs, M. W. (2019, Mayo 11). Developer mozilla. Retrieved from https://developer.mozilla.org/es/docs/Learn/JavaScript/First_steps/Qu\%C3\%A9_es _JavaScript 
Docs, M. W. (2019, Septiembre 10). Developer mozilla. Retrieved from https://developer.mozilla.org/es/docs/Web/CSS

Miyahira Arakak, J. (1995). El arbitraje editorial en las revistas médicas. Revista Medica Herediana, Recuperado en 27 de noviembre de 2020, de http://www.scielo.org.pe/scielo.php?script=sci_arttext\&pid=S1018130X1995000300001\&lng=es\&tlng=es. .

Php. (2001, Septiembre 18). Php. Retrieved from https://www.php.net/manual/es/introwhatis.php

Rennie, D. (1998). The present state of medical journals. The Lancet, 22.

Silva AyÇaguer, L. C. (2011). El arbitraje de las revistas médicas, la gestión editorial en red y la calidad de la publicación científica . ACIMED, 91-93.

Szklo , M., \& Nieto , F. (1993). El papel de las revistas de salud pública. San Hig Púb, 331334.

Wordpress. (2000). Retrieved from Wordpress.com: https://es.wordpress.com/

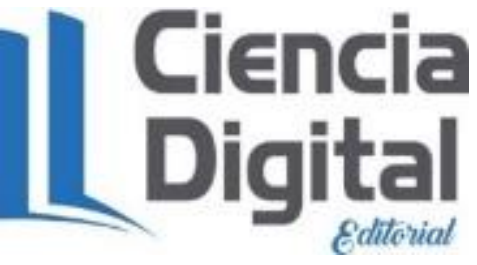




\section{Para citar el artículo indexado}

Guerra Poalasin, D. O. . (2021). Implementación de una aplicación informática para la verificación de parámetros de publicación de un artículo científico en revistas indexadas. AlfaPublicaciones, 3(2), 25-41. https://doi.org/10.33262/ap.v3i2.42

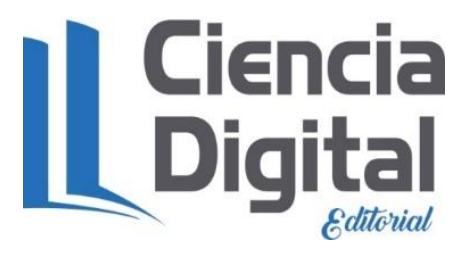

El artículo que se publica es de exclusiva responsabilidad de los autores y no necesariamente reflejan el pensamiento de la Revista Alpha Publicaciones.

El artículo queda en propiedad de la revista y, por tanto, su publicación parcial y/o total en otro medio tiene que ser autorizado por el director de la Revista Alpha Publicaciones.
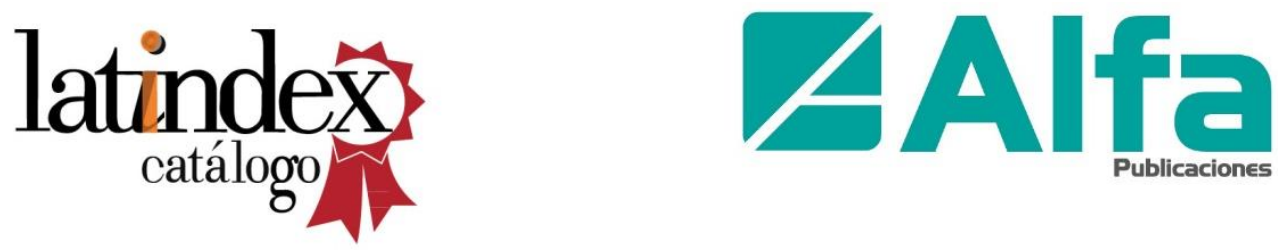